\title{
Barrier Function Nonlinear Optimization for Optimal Decompression of Divers
}

\author{
Le Feng, Christian R. Gutvik, Tor A. Johansen, Dan Sui
}

\begin{abstract}
This paper is based on a comprehensive dynamic mathematical model (Copernicus) of vascular bubble formation and growth during and after decompression from a dive. The model describes the underlying relationship between Venous Gas Emboli (VGE) and risk of severe Decompression Sickness (DCS). By using the Copernicus model the diving decompression problem can be formulated as a nonlinear optimal control problem, where the objective is to minimize the total ascend time subject to constraints on the maximum number of bubbles in the pulmonary artery (also referred to as the decompression stress). A recent study reveals that the optimal solution can be obtained by solving the optimization problem with some equality constraints. Inspired by which, a simpler approach using barrier function is proposed in this paper, through which we achieve a more efficient and robust numerical implementation. The paper also studies the effect of ascent profile parameterization.
\end{abstract}

Keywords: nonlinear model predictive control; decompression sickness; barrier function; dynamic decompression model.

\section{INTRODUCTION}

One of the major causes of decompression sickness (DCS) is the accumulated inert gas during a dive and the ambient pressure reduction during ascent after the dive. To prevent this and safely ascend a diver, many models and decompression schedules have been proposed. Most of them are based on the principles described in [1], i.e., set up a sufficiently large gradient for gas elimination and assume that substantial supersaturation can be tolerated without significant bubble formation. An alternative model was proposed in 1970 by Hills [2], where bubble formation would happen as soon as supersaturation was present, suggesting that bubble formation would occur early in the Haldanean type models and that long decompressions from severe dives would be needed to allow their elimination.

One limitation of these procedures is that they are evaluated using clinical symptoms of DCS as an endpoint, while the underlying mechanisms have been ignored. However the symptomatology of DCS is diffuse and there are ethical concerns evaluating procedures through provoking DCS on the test subjects. In 2008, Gutvik et. al. proposed a dynamic 2-phase model for vascular bubble formation [3]. This model assumes that there is a close relationship between VGE

Le Feng, Tor A. Johansen and Dan Sui are with Department of Engineering Cybernetics, Norwegian University of Science and Technology, E-mail: \{feng.le,tor.arne.johansen, sui.dan\}@itk.ntnu.no.

Christian R. Gutvik is with Department of Circulation and Imaging, Norwegian University of Science and Technology, Email:christian.gutvik@ntnu.no.

This work has been supported by UWATEC AG, Switzerland, by The Research Council of Norway and the Norwegian Petroleum Directorate Norsk Hydro, Esso Norge and Statoil under the "dive contingency contract" (no 4600002328) with Norwegian Underwater Intervention (NUI). and risk of severe DCS and VGE can be detected using ultrasonic Doppler and imaging. Later a statistical correlation between VGE and DCS has been reported [4] where it is also shown that existing decompression algorithms (standard diving tables and protocols) are not consistent in terms of controlling the VGE formation and consequently the risk of DCS.

With the proposed dynamic mathematical model we can numerically optimize the decompression profile by minimizing the total ascend time subject to constraints on the maximum peak decompression stress. Intuitively the optimal solution is on the boundary of the feasible region, i.e., when the decompression stress constraints are activated. Therefore in a recent study [5], [6], Gutvik et al. solves the optimization problem by minimizing the total ascend time subject to an equality constraint (the maximum stress during ascent equals the pre-defined threshold). There are some limitations in this approach. For example, decompression stress of some "simple dives" is always less than the threshold hence will not provoke the equality constraint. Secondly after extreme dives it may be impossible to avoid that the stress exceeds the threshold. Consequently the method will rely on some extra logic to handle such cases. Furthermore, there are some numerical challenges to implement the optimization since we do not know in advance the point in time when the maximum stress is reached. Considering these limitations we propose a barrier function nonlinear optimization approach [11] in this paper through which an efficient and numerically more robust implementation is achieved.

A further objective in this paper is to systematically study what is the effect and performance loss by various ascend trajectory parameterizations. Generally the theoretically optimal solution is a continuous time-depth function which is not only computationally inefficient for numerical implementation in a low-cost diving computer but also not practical for a diver to follow. Thus different parameterization methods are studied to address such problems.

In the following sections we will demonstrate how optimal decompression profiles are calculated using optimal control. Since the physiological state of the diver changes continuously, the dynamic model should be updated based on present measurements, and the ascend profile should be re-optimized at regular intervals in a receding horizon fashion in a diving computer. Although we adopted several methodologies to reduce the complexity, the current dynamic model-based optimal diving decompression strategy is still preliminary and of more conceptual nature. The main obstacle prevents it from direct implementation on a low- 
cost diving computer is the prohibitive amount of floating point numerical computations compared to the limited CPU capacity and power consumption requirements. Therefore an (approximate) explicit solution of nonlinear constrained optimization using multi-parametric nonlinear programming [7], [8] is promising and suited for this type of problem. This direction is taken in [5], [9] and pursued further in our ongoing work.

The paper is organized as follows: after introduction a brief description of the dynamic model is given in Section II. The optimal decompression problem is formulated and solved in Section III followed by numerical results presented in Section IV. Conclusions are given in Section V.

\section{MOdEL DESCRIPTION}

\section{A. Nomenclature ( [3], [4])}

\begin{tabular}{|c|c|c|c|}
\hline Param. & Description & Value & Unit \\
\hline$n_{t}$ & Number of tissues. & 2 & - \\
\hline$D$ & Bubble barrier diffusivity. & 20 & $\mu m^{2} / \mathrm{min}$ \\
\hline$\alpha_{b}$ & Blood solubility. & 0.0158 & $\mathrm{msw}^{-1}$ \\
\hline$h$ & Bubble barrier thickness. & 0.1 & $\mu m$ \\
\hline$\gamma$ & Surface tension. & 17.890 & $\mu m \mathrm{msw}$ \\
\hline$P_{\text {meta }}$ & $\begin{array}{l}\text { Partial pressure of the metabolic } \\
\text { gases. }\end{array}$ & 0.1773 & atm \\
\hline$c_{s}$ & Coefficient. & 0.9479 & $\mu m^{3}$ atm \\
\hline$\varepsilon_{\tau, 1}$ & $\begin{array}{l}\text { Time constant correction of mus- } \\
\text { cles. }\end{array}$ & 0.2868 & - \\
\hline$\varepsilon_{\tau, 2}$ & Time constant correction of fat. & 0.8115 & - \\
\hline$\alpha_{t, 1}$ & Tissue solubility of muscles. & 0.0278 & $\operatorname{atm}^{-1}$ \\
\hline$\alpha_{t, 2}$ & Tissue solubility of fat. & 0.0640 & $\operatorname{atm}^{-1}$ \\
\hline$\delta$ & Bubble nuclei density. & 0.0000005 & $\# / \mu m^{3}$ \\
\hline$k_{m}$ & Measurement gain. & 200 & - \\
\hline$V_{0}$ & Dead volume for detection. & 0.005 & $\begin{array}{l}\text { \#bubbles } \\
/ \mathrm{cm}^{2} / \mathrm{min}\end{array}$ \\
\hline$V_{1}$ & Tissues volume of muscles. & 28.4 & $\mathrm{dm}^{3}$ \\
\hline$V_{2}$ & Tissues volume of fat. & 11.7 & $\mathrm{dm}^{3}$ \\
\hline$r_{i}$ & Bubble radius in tissue $i$. & & $\mu m$ \\
\hline$p_{t, i}$ & Gas tension in tissue $i$. & & msw \\
\hline$P_{a m b}$ & Ambient pressure. & & msw \\
\hline$\dot{P}_{a m b}$ & Rate of descent. & & $\mathrm{msw} / \mathrm{min}$ \\
\hline$f_{N_{2}}$ & $\begin{array}{l}\text { Fraction of nitrogen in inspired } \\
\text { gas. }\end{array}$ & 0.78 & - \\
\hline$\omega_{1, \text { dive }}$ & Blood perfusion of muscles (dive). & 0.16 & $\mathrm{~L} / \mathrm{min} / \mathrm{dm}^{3}$ \\
\hline$\omega_{1, \operatorname{surf}}$ & $\begin{array}{l}\text { Blood perfusion of muscles (sur- } \\
\text { face). }\end{array}$ & 0.091 & $\mathrm{~L} / \mathrm{min} / \mathrm{dm}^{3}$ \\
\hline$\omega_{2, \text { dive }}$ & Blood perfusion of fat (dive). & 0.047 & $\mathrm{~L} / \mathrm{min} / \mathrm{dm}^{3}$ \\
\hline$\omega_{2, \text { surf }}$ & Blood perfusion of fat (surface). & 0.043 & $\mathrm{~L} / \mathrm{min} / \mathrm{dm}^{3}$ \\
\hline$y$ & $\begin{array}{l}\text { Measurement of the number of } \\
\text { bubbles in the pulmonary artery. }\end{array}$ & & $\begin{array}{l}\text { \#bubbles } \\
/ \mathrm{cm}^{2} / \mathrm{min}\end{array}$ \\
\hline
\end{tabular}

\section{B. Dynamic model}

The dynamic model introduced in [3] and [4] is presented here for ease of reading. The diver can be regarded as a dynamic system where the state vector $x$ is influenced by the input vector $u$. Copernicus is a nonlinear model giving the bubble dynamics:

$$
\begin{aligned}
& \dot{x}=f(x, u) \\
& y=h_{m}(x, u)
\end{aligned}
$$

where

$$
\begin{aligned}
& f(x, u)=\left[\begin{array}{c}
\frac{D \alpha_{b}}{h}\left(x_{2 i}-x_{2 n_{t}+1}-\frac{2 \gamma}{x_{2 i-1}}+P_{m e t a}+\frac{c_{s}}{x_{2 i-1}^{3}}\right)-\frac{x_{2 i-1}}{3} u_{1} \\
x_{2 n_{t}+1}+\frac{4 \gamma}{3 x_{2 i-1}}-P_{m e t a} \\
\varepsilon_{\tau, i} \frac{\alpha_{b}}{\alpha_{t, i}}\left(u_{2} x_{2 n_{t}+1}-x_{2 i}\right) u_{2+i}+\dot{p}_{r, i}(x) \\
\vdots \\
\vdots \\
u_{1}
\end{array}\right], \\
& h_{m}(x, u)=\frac{4 \pi}{3} \delta k_{m} \sum_{i=1}^{n_{t}} r_{i}^{3} V_{i} \omega_{i}-V_{0}, \\
& x=\left[\begin{array}{llllllll}
r_{1} & p_{t, 1} & r_{2} & p_{t, 2} & \ldots & r_{n_{t}} & p_{t, n_{t}} & P_{a m b}
\end{array}\right]^{T}, \\
& u=\left[\begin{array}{llll}
\dot{P}_{a m b} & f_{N_{2}} & \omega_{1} & \omega_{2}
\end{array}\right]^{T},
\end{aligned}
$$

$$
\begin{aligned}
\dot{p}_{r_{i}}(x)= & -\frac{4 \pi D \alpha_{b} \delta}{h \alpha_{t, i}} x_{2 i-1}^{2}\left(x_{2 i}-x_{2 n_{t}+1}-\frac{2 \gamma}{x_{2 i-1}}\right. \\
& \left.+P_{\text {meta }}+c_{s} \frac{1}{x_{2 i-1}^{3}}\right) .
\end{aligned}
$$

Please note that $\dot{p}_{r_{i}}(x) \approx 0$ referring to [4], since the amount of gas molecules dissolved in the tissues is believed to be much larger than in free gas phase. Equation (1d) gives the measurement of the number of bubbles detected in the pulmonary using ultrasonic image scanning [10].

The model is constituted of $n_{t}$ tissues. Theoretically $n_{t}$ can be chosen as large as we want therefore the model can approximate a human body as precisely as needed. On the other hand it's convenient to keep $n_{t}$ as small as possible considering the size of the resulting optimization problem. In this paper we use two tissues $(i=\{1,2\})$, i.e., the muscles and fat tissues, which represents the dynamics of fast and slow tissues, respectively [4], [5].

\section{OPTIMAL DECOMPRESSION PROBLEM}

\section{A. Parameterization of the decompression profile}

The main purpose of the optimal decompression is to ascend a diver as fast as possible while the number of bubbles in the pulmonary $y$ (also referred to as the decompression stress) does not exceed a pre-defined threshold. Theoretically the optimal solution is a continuous time-depth profile, which in practice has some limitation to implement. For example, it's hard to numerically solve the optimal continuous timedepth profile [5] and it's also hard for a diver to follow such a continuous ascending profile. Therefore in [5], [6], [9] a stepwise decompression formulation is proposed, illustrated in Figure 1.

Common diving protocols assume a fixed number of stopping depths $\left(\rho_{1}, \rho_{2}, \ldots, \rho_{n_{s}}\right)$. Assuming the ascending speed of the diver $(a)$ is constant, the time for ascending $\Delta t_{a, 1}+\Delta t_{a, 2}+\ldots+\Delta t_{a, \text { surf }}$ is a constant. Suppose $\Delta t_{1}, \Delta t_{2}, \ldots, \Delta t_{n_{s}}$ are the stopping times on each stopping 


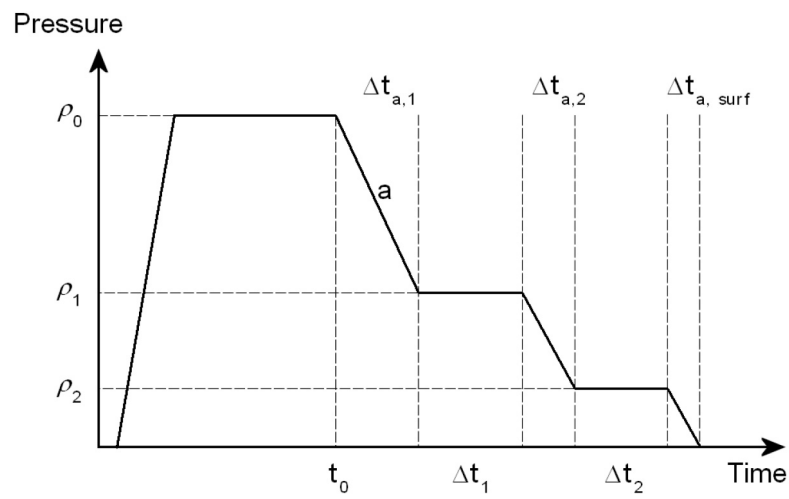

Fig. 1. Stepwise decompression profile

depth. Considering the main objective of optimal ascent, our goal is to

$$
\min \sum_{i=1}^{n_{s}} \Delta t_{i}
$$

With such parameterization there are $n_{s}$ parameters to be optimized subject to $\Delta t_{i} \geqslant 0$. However in some cases, e.g., a long deep dive, there shall be quite many stopping depths to safely ascend a diver. In other words, $n_{s}$ can still be large and the computational effort can still be huge. In addition, this parameterization tends to give a "flat minimum" with associated numerical challenges. This motivated another parameterization method used in [5] and studied in this paper, illustrated in Figure 2.

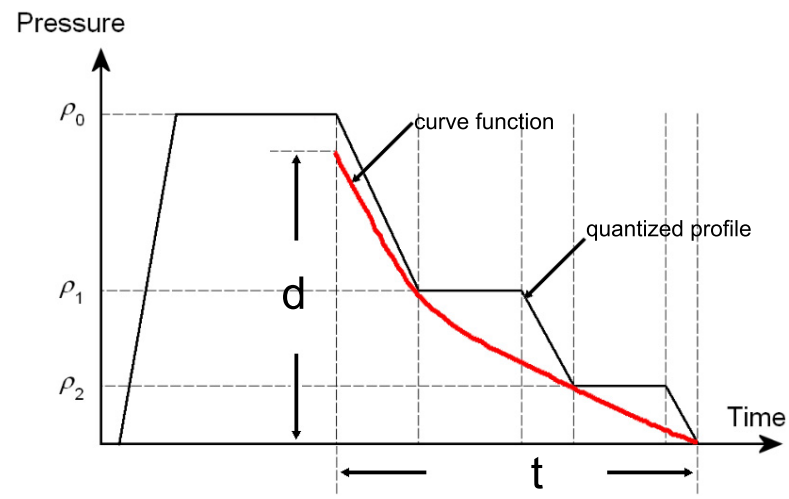

Fig. 2. Curve decompression profile

The idea is to first define a curve function with two parameters $d$ and $t$ (illustrated in Figure 2). For a given $d$ and $t$, the curve can be quantized and generate the stepwise formulation and also a total ascend time according to stopping depths $\left(\rho_{1}, \rho_{2}, \ldots, \rho_{n_{s}}\right)$. Then instead of optimizing $n_{s}$ parameters in the original formulation, now only two parameters need to be optimized. Obviously this parameterization and quantization method leads to a simplified optimization problem but may also introduces some conservatism, that is, the total ascending time $(t)$ may be longer and/or the stress threshold may be exceeded. The benefit and performance loss of this parameterization method will be studied by simulations in
Section IV. For detailed information of the curve function please refer to [5].

\section{B. Cost function}

First we will give the cost function for the stepwise parameterization method. The optimization variable vector is defined as

$$
z=\left[\Delta t_{1}, \Delta t_{2}, \ldots, \Delta t_{n_{s}}\right] .
$$

Then the cost function is

$$
V(z)=c^{T} z,
$$

where $c=[1,1, \ldots, 1]$.

Please note that for curve parameterization method the cost function is the same but $z$ is not the optimizer. Instead, only $d$ and $t$ are the optimizers. From a pre-defined curve function $\left(z=q_{t}(d, t)\right)$ and a set of pre-defined possible stopping depths $\left(\rho_{1}, \rho_{2}, \ldots, \rho_{n_{s}}\right), z$ can be calculated and used in the cost function.

\section{Constraints}

The two parameterization methods share same constraints, which is nonlinear and in a receding horizon manner. That is, the output $y$ (decompression stress) at not only current time but also during the predicted horizon shall not exceed the limits. Mathematically, the nonlinear constraints can be written as

$$
H(z)=\left[\begin{array}{c}
y\left(x_{1}\right)-y_{\text {limit }} \\
y\left(x_{2}\right)-y_{\text {limit }} \\
\vdots \\
y\left(x_{n_{k}}\right)-y_{\text {limit }}
\end{array}\right] \leqslant 0 .
$$

where the future state $x_{i}$ is predicted through numerical integration of the model and $i \in\left\{1,2, \ldots, n_{k}\right\}$ are the discretization times.

\section{Model discretization and integration}

We use Euler integration to implement the model discretization and integration, that is:

$$
x_{k+1}=x_{k}+\Delta T_{i} f\left(x_{k}, u_{k}\right) .
$$

Note that $\Delta T_{i}$ is the integration step size where the subscript $i$ means the integration step size is not constant over the prediction horizon. The reason for this is to obtain a continuously differentiable constraint function. The optimization vector $z$ contains the time of the decompression stops, hence the integration step size will be dependent of $z$. If we keep the integration step size constant, the number of integration steps $n_{k}$ will be a function of $z$. This would have made the constraint function discontinuous in $z$. As described in [9], we have the following integration step sizes.

During the ascents:

$$
\Delta T_{a, i}=\frac{\rho_{i}-\rho_{i-1}}{a \cdot n_{\text {ascent }, i}}, \quad i=1,2, \cdots n_{s}+1
$$

where $n_{\text {ascent }, i}$ is the number of integration steps from bottom to the first decompression stop, between the stops and from the last stop to the surface. 
During the decompression stops:

$$
\Delta T_{s, i}=\frac{z_{i}}{n_{\text {stop }}}, \quad i=1,2, \cdots n_{s}
$$

where $n_{\text {stop }}$ is the number of integration steps during the decompression stops.

After reaching the surface:

$$
\Delta T_{\text {surf }}=\frac{\Delta t_{\text {surf }}}{n_{\text {surf }}}
$$

where $\Delta T_{\text {surf }}$ is the stopping time at the surface and $n_{\text {surf }}$ is the number of integration steps at the surface.

\section{E. Barrier function}

In constrained optimization, a barrier function is a continuous function whose value on a point increases to infinity as the point approaches the boundary of the feasible region. It is used as a penalizing term for violations of constraints [11]. The two most popular types of barrier functions are inverse barrier functions and logarithmic barrier functions. Generally constrained nonlinear optimization is computational expensive. Usage of barrier functions can reduce the computational complexity. The cost functions with the two types of barrier functions are given below

$$
\begin{array}{rr}
\text { inverse : } & \sum_{i=1}^{n_{s}} \Delta t_{i}+\sum_{i=1}^{n_{k}} \frac{\mu}{y_{\text {limit }}-y\left(x_{i}\right)} \\
\text { logarithmic : } & \sum_{i=1}^{n_{s}} \Delta t_{i}-\mu \sum_{i=1}^{n_{k}} \log \left(y_{\text {limit }}-y\left(x_{i}\right)\right)
\end{array}
$$

where $\mu$ is called barrier parameter.

In typical barrier function algorithms [11], the sequence of barrier parameters $\left\{\mu_{1}, \mu_{2}, \ldots\right\}$ must converge to zero so that, as more and more iterations are made, we recover the solution of the original nonlinear programming problem. If $\mu$ is decreased too slowly, a large number of iterations will be required for convergence; but if it is decreased too quickly, the progress of the iterations may be slowed down [11]. In this paper we use a fixed barrier parameter, that is, $\mu_{1}=\mu_{2}=\cdots=\mu$.

The motivation for this simplification is that the model is uncertain and the threshold $y_{\text {limit }}$ is uncertain, such that an approximate solution is sufficient. It is considered more important to reduce computation time as long as the errors are within safety margins.

\section{F. Optimization problem}

We can see that the optimal decompression problem can be expressed as follows. For stepwise formulation:

$$
\min _{z} \sum_{i=1}^{n_{s}} \Delta t_{i}
$$

s.t.

$$
\begin{array}{r}
-z \leqslant 0 \\
H(z) \leqslant 0
\end{array}
$$

For curve formulation:

$$
\min _{t, d} \sum_{i=1}^{n_{s}} \Delta t_{i}
$$

s.t.

$$
\begin{array}{r}
-z \leq 0 \\
H(z) \leq 0
\end{array}
$$

For inverse barrier function:

$$
\min _{t, d}\left(\sum_{i=1}^{n_{s}} \Delta t_{i}+\sum_{i=1}^{n_{k}} \frac{\mu}{y_{\text {limit }}-y\left(x_{i}\right)}\right)
$$

s.t.

$$
-z \leqslant 0
$$

For logarithmic barrier function:

$$
\min _{t, d}\left(\sum_{i=1}^{n_{s}} \Delta t_{i}-\mu \sum_{i=1}^{n_{k}} \log \left(y_{\text {limit }}-y\left(x_{i}\right)\right)\right.
$$

s.t.

$$
-z \leqslant 0
$$

The optimization problem is solved as follows. The diving computer first measures the ambient pressure $P_{a m b}$ and heart rate; then estimates a current state of the diver based on this measurement, manual input, and a mathematical model; uses this information to generate an optimal ascending profile for the diver (implemented in Matlab with the TOMLAB 'ucsolve' algorithm using a Quasi-Newton method BFGS). The diver tries to follow the command he received and ascend to a stopping depth as suggested (which might not be very precise). The diving computer measures a new ambient pressure $P_{a m b}$ and heart rate and solves the optimization problem repetitively at this new time step.

\section{RESUlTS}

In this section a two-tissue model is adopted, namely, muscle tissue and fat tissue. Based on this model we will demonstrate the benefits of the barrier function implementation and also study the effect of the parameterization methods.

Four dives are simulated to evaluate the four approaches, namely, equality constrained stepwise optimization, equality constrained curve optimization [5], logarithm barrier function optimization and inverse barrier function optimization. We noticed that an accurate initialization of the solver has much more impact on the stepwise optimization than on the curve optimization. Therefore we can initialize the stepwise optimization with the solution of the the curve optimization. The possible stopping depths are pre-defined to be $[21,18,15,12,9,6,3]$ with $n_{s}=7$. Then the prediction horizon $n_{k}$ can be calculated by $n_{k}=n_{s} \cdot n_{\text {stop }}+n_{\text {surf }}+\sum_{i=1}^{n_{s}+1} n_{\text {ascend }, i}$. The dive schedule is given in a vector form: the first element means the diving depth, the second element is bottom time while the third is heart rate and fourth element means the fraction of nitrogen in the inspired gas. The simulation results are given in the following tables where $t_{\text {opt }}$ is the optimal 
ascending time and $d_{o p t}$ is the other optimizer in Figure 2, $t_{c p u}$ is the computational time which is obtained on an Intel Due $2.53 \mathrm{G}$ laptop (unit for time is minute).

TABLE I

EQUALITY CONSTRAINED STEPWISE OPTIMIZATION

\begin{tabular}{ccc}
\hline Schedule & \multicolumn{2}{c}{ Equality constrained stepwise optimization } \\
\cline { 2 - 3 } & $t_{\text {opt }}$ & $t_{c p u}$ \\
\hline$[45,25,95,0.78]$ & 55.0201 & 4.8846 \\
{$[35,25,95,0.78]$} & 24.4727 & 2.6281 \\
{$[55,20,90,0.78]$} & 47.9364 & 4.0528 \\
{$[55,20,95,0.78]$} & 60.4171 & 5.9422 \\
\hline
\end{tabular}

TABLE II

EQUALITY CONSTRAINED CURVE OPTIMIZATION

\begin{tabular}{cccc}
\hline Schedule & \multicolumn{3}{c}{ Equality constrained curve optimization } \\
\cline { 2 - 4 } & $t_{\text {opt }}$ & $d_{\text {opt }}$ & $t_{c p u}$ \\
\hline$[45,25,95,0.78]$ & 62.7012 & 18.8343 & 0.5245 \\
{$[35,25,95,0.78]$} & 27.0753 & 12.2132 & 0.5091 \\
{$[55,20,90,0.78]$} & 54.7797 & 17.0896 & 0.5205 \\
{$[55,20,95,0.78]$} & 69.6830 & 20.0976 & 0.5133 \\
\hline
\end{tabular}

TABLE III

LOGARITHM BARRIER FUNCTION

\begin{tabular}{|c|c|c|c|c|c|c|}
\hline \multirow[t]{3}{*}{ Schedule } & \multicolumn{6}{|c|}{ Logarithm barrier function } \\
\hline & \multicolumn{3}{|c|}{$\mu=0.1$} & \multicolumn{3}{|c|}{$\mu=0.01$} \\
\hline & $t_{\text {opt }}$ & $d_{o p t}$ & $t_{c p u}$ & $t_{o p t}$ & $d_{o p t}$ & $t_{c p u}$ \\
\hline$[45,25,95,0.78]$ & 62.80 & 18.84 & 0.46 & 62.71 & 18.83 & 1.78 \\
\hline$[35,25,95,0.78]$ & 27.18 & 12.23 & 0.18 & 27.09 & 12.21 & 1.04 \\
\hline$[55,20,90,0.78]$ & 54.88 & 17.10 & 0.27 & 54.79 & 17.09 & 2.70 \\
\hline$[55,20,95,0.78]$ & 69.78 & 20.10 & 0.10 & 69.69 & 20.10 & 1.50 \\
\hline
\end{tabular}

TABLE IV

INVERSE BARRIER FUNCTION

\begin{tabular}{|c|c|c|c|c|c|c|}
\hline \multirow[t]{3}{*}{ Schedule } & \multicolumn{6}{|c|}{ Inverse barrier function } \\
\hline & \multicolumn{3}{|c|}{$\mu=0.001$} & \multicolumn{3}{|c|}{$\mu=0.01$} \\
\hline & $t_{o p t}$ & $d_{o p t}$ & $t_{c p u}$ & $t_{\text {opt }}$ & $d_{o p t}$ & $t_{c p u}$ \\
\hline$[45,25,95,0.78]$ & 62.95 & 18.85 & 0.18 & 63.49 & 18.88 & 0.13 \\
\hline$[35,25,95,0.78]$ & 27.31 & 12.24 & 0.16 & 27.81 & 12.31 & 0.15 \\
\hline$[55,20,90,0.78]$ & 55.03 & 17.11 & 0.18 & 55.56 & 17.14 & 0.13 \\
\hline$[55,20,95,0.78]$ & 69.94 & 20.11 & 0.14 & 70.49 & 20.14 & 0.13 \\
\hline
\end{tabular}

From Table I and II we can see the effect of input trajectory parameterization. The stepwise parameterization gives shorter ascending time (from $10.49 \%$ to $15.35 \%$ ) at a cost of much longer computational time (from $516 \%$ to $1158 \%$ ).

From II to IV we can see that the barrier function approach may lead to conservatism, i.e., the optimal ascending time is slightly longer, depending on $\mu$ and barrier function. However such conservatism with respect to the constraint is not always a bad thing. Since the value of $y_{\text {limit }}$ and model parameters are uncertain, barrier function method adds a safety margin. Using inverse barrier function can reduce the computational time by more than $80 \%$. We shall also notice that for logarithm barrier function, a too small value of $\mu$ can cause too much computational time.

In the following the simulation results are given in Figure 3 to Figure 6. The sub-figure on the top of each figure gives the time-depth decompression profile while the bottom one is the output $y$ (measurement of the number of bubbles in the pulmonary artery). The black solid line corresponds to the equality constrained curve optimization method. The black dash-dotted lines correspond to the proposed logarithm/inverse barrier function methods $(\mu=0.01)$. However the results are very close to the equality constrained curve optimization method which makes them not distinguishable in the figures. The blue dashed line describes the equality constrained stepwise optimization method while the red dotted line in the bottom sub-figure is the stress threshold which is designed not to be violated during simulations.
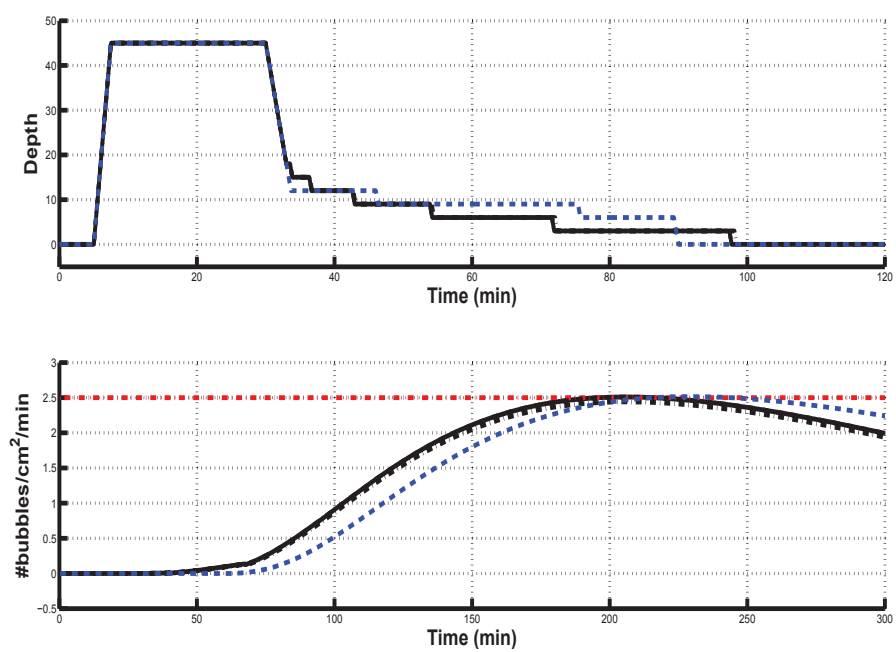

Fig. 3. Decompression profiles of diving schedule $[45,25,95,0.78]$
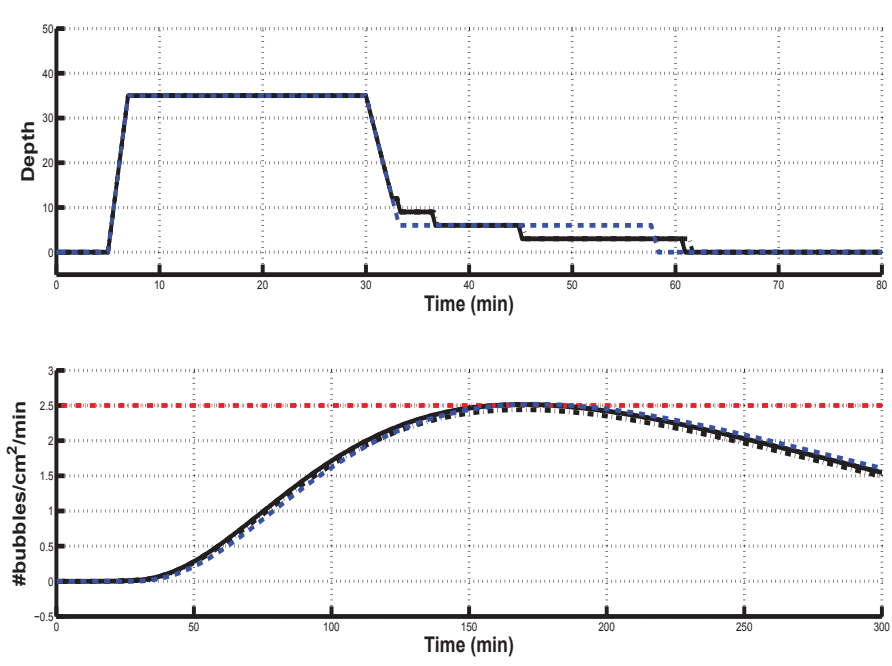

Fig. 4. Decompression profiles of diving schedule $[35,25,95,0.78]$ 

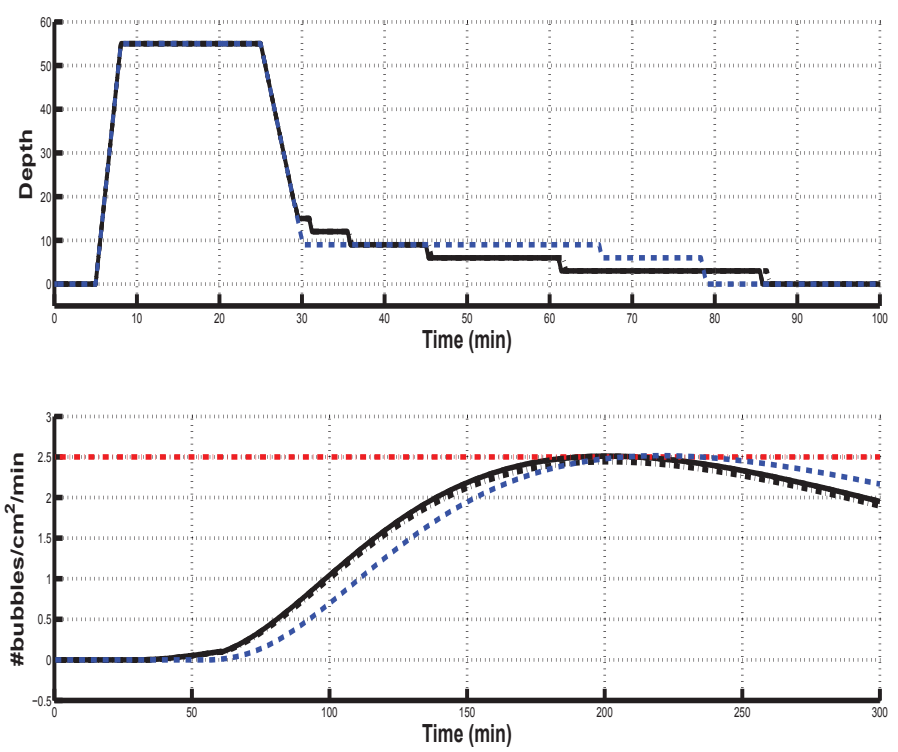

Fig. 5. Decompression profiles of diving schedule [55, 20, 90, 0.78]
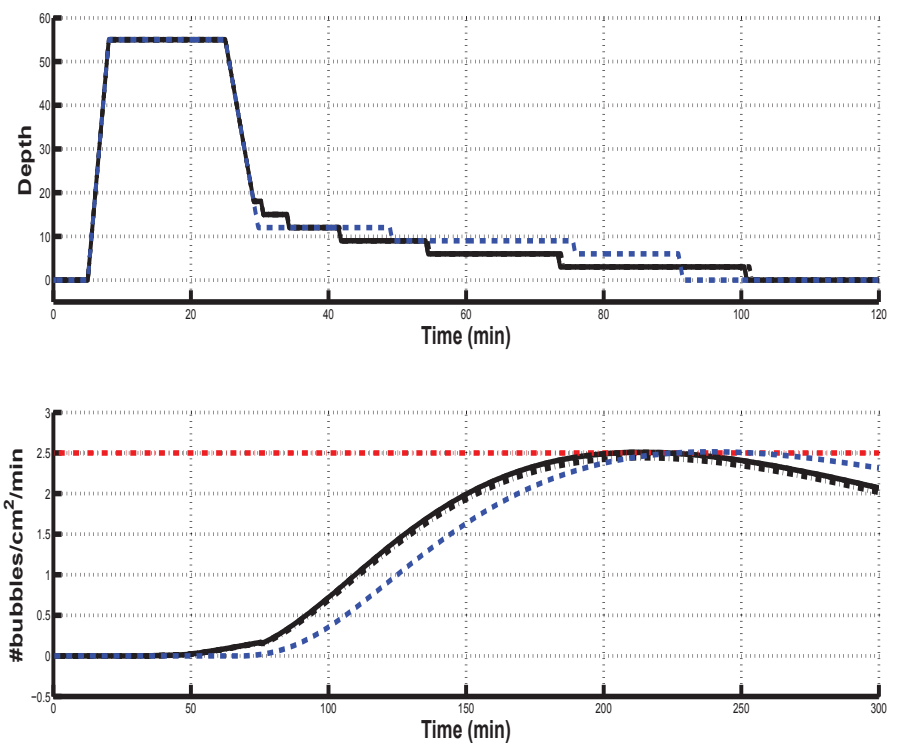

Fig. 6. Decompression profiles of diving schedule $[55,20,95,0.78]$

From these figures one may conclude that all these four methods respect the stress constraint. The stepwise solution gives shortest ascending time but at a high computational cost. Curve parameterization significantly reduces the computational cost with an acceptable performance degradation and has proven to be much less sensitive to an accurate initialization of the solver, as the stepwise parameterization is more prone to local minima. Using barrier function to substitute the equality constraint leads to a more efficient numerical implementation and the associated conservatism is neglectable according to the simulation results.

\section{CONCLUSION AND FUTURE WORK}

In this paper we applied a new dynamic model on bubble formulation and evolution in human body. The model is based on Venous Gas Emboli (VGE) other than decompression sickness (DCS). Based on this model the decompression problem is formulated as an optimal control problem, i.e., minimizing the total ascending time subject to safety constraints. Inverse barrier function and logarithmic barrier function are adopted in the nonlinear optimization problem. Simulation is carried on a two tissue model and the results are promising.

The optimization requires a large amount of floating point numerical computations. This is considered prohibitive for implementation in a low-cost diving computer with limited CPU capacity and power consumption requirements. Therefore in the future it is planned to work towards explicit solutions, using methods described in [7]-[9]. Another work in the future is to apply quadratic barrier function which can handle infeasibility problem during optimization.

\section{REFERENCES}

[1] A. E. Boycott, C. C. Damant, and J. S. Haldane, "The prevention of compressed - air illness," Journal of Hygiene, vol. 8, no. 342-443, 1908.

[2] B. A. Hills, "Vital issues in computing decompression schedules from fundamentals i. critical supersaturation versus phrase equilibration," Int J Biometeror, vol. 14, no. 2, pp. 111-128, 1970.

[3] C. R. Gutvik and A. O. Brubakk, "A dynamic 2-phase model for vascular bubble formation during decompression of divers," IEEE Transactions Biomedical Engineering, 2009.

[4] C. R. Gutvik, R. G. Dunford, Z. Dujic, and A. O. Brubakk, "Parameter estimation of the copernicus decompression model using non-linear optimization," Medical and Biological Engieering and Computing, 2009.

[5] C. R. Gutvik, T. A. Johansen, and A. O. Brubakk, "Nonlinear optimization of a 2-phase decompression model and an approximate, explicit solution," In preparation, 2009.

[6] C. R. Gutvik and A. O. Brubakk, "Optimal decompression profiles based on nonlinear dynamic bubble models," in Annual Meeting Abstracts. Undersea and Hyperbaric Medicine Society, 2005.

[7] A. Grancharova, T. A. Johansen, and P. Tøndel, "Computational aspects of approximate explicit nonlinear model predictive control," in Assessment and Future Directions of Nonlinear Model Predictive Control, ser. Lecture Notes in Control and Information Sciences, R. Findeisen, F. Allgöwer, and B. L., Eds. Springer Berlin / Heidelberg, 2007, vol. 358, pp. 181-192.

[8] T. A. Johansen, "Approximate explicit receding horizon control of constrained nonlinear systems," Automatica, vol. 40, pp. 293-300, 2004.

[9] P. Tøndel, C. R. Gutvik, and T. A. Johansen, "Optimal decompression - diving computer implementation aspects of an explicit solution," Department of Engineering Cybernetics, Norwegian University of Science and Technology, Tech. Rep., 2005.

[10] A. Brubakk and O. Eftedal, Evaluation of reverse dive profiles, Smithsonian Institution, Washington DC, 2000, ch. Reverse dive profiles, pp. $111-121$.

[11] J. Nocedal and S. J. Wright, Numerical optimization. Springer, 2006. 